\title{
Segmentation of Cardiac Structures Simultaneously from Short- and Long-Axis MR Images
}

\author{
Juha Koikkalainen ${ }^{1}$, Mika Pollari ${ }^{1}$, Jyrki Lötjönen ${ }^{2}$, Sari Kivistö ${ }^{3}$, and \\ Kirsi Lauerma ${ }^{3}$ \\ 1 Laboratory of Biomedical Engineering, Helsinki University of Technology, \\ P.O.B. 2200, FIN-02015 HUT, Finland \\ \{Juha.Koikkalainen, Mika.Pollari\}@hut.fi \\ 2 VTT Information Technology, P.O.B. 1206, FIN-33101 Tampere, Finland \\ Jyrki.Lotjonen@vtt.fi \\ 3 Helsinki Medical Imaging Center, University of Helsinki, P.O.B. 281, FIN-00029 \\ HUS, Finland
}

\begin{abstract}
We introduce a framework for the automatic segmentation of the ventricles, atria, and epicardium simultaneously from cardiac magnetic resonance (MR) volumes. The basic idea is to utilize both shortaxis (SA) and long-axis (LA) MR volumes. Consequently, anatomical information is available from the whole heart volume. In this paper, the framework is used with deformable model based registration and segmentation methods to segment the cardiac structures. A database consisting of the cardiac MR volumes of 25 healthy subjects is used to validate the methods.

The results presented in this paper prove that by using both the SA and LA MR volumes the ventricles and atria can be simultaneously segmented from cardiac MR volumes with a good accuracy. The results show that notably better segmentation results are obtained when the LA volumes are used in addition to the SA volumes. For example, this enables accurate segmentation of the ventricles also in the basal and apical levels.
\end{abstract}

\section{Introduction}

Cardiac MR imaging provides accurate information on the anatomy of the heart. This information can be used to study and analyze the cardiac function [1. Segmentation of cardiac structures is a pre-requisite for the determination of quantitative measurements, such as the volume of the ventricles, wall thickness, or ejection fraction. Automated segmentation algorithms are needed to produce objective, reproducible segmentations, and to avoid the need for the time-consuming manual segmentation of large amount of data.

Cardiac MR volumes have often low quality: the signal is lost due to the blood flow and partial volume effect, and the volumes are noisy and corrupted with artifacts. Therefore, a priori knowledge is usually utilized in segmentation. In model-based segmentation, for example, atlases or statistical models can be 
used. In [2], an anatomical atlas was constructed from a set of healthy subjects, and the atlas was used to give an a priori estimate in the non-rigid registrationbased segmentation of the ventricles. In [3], a probabilistic anatomical atlas was used to initialize the parameters of expectation maximization algorithm. Statistical shape (Active Shape Model, ASM) 4 and appearance (Active Appearance Model, AAM) [5] models are popular methods in cardiac segmentation. Kaus et al. [6] used a statistical shape model to regularize the deformable model-based segmentation of the left ventricle. In [7], Mitchell et al. combined ASM and AAM approaches in the cardiac segmentation, and in [8], they presented fully 3D AAM algorithm for the same problem.

Most of the recent cardiac segmentation studies using MR images deal with the segmentation of the left ventricle and epicardium 6 89,10]. In some studies, also the right ventricle is segmented [2/37/1]. These studies have been done using short-axis MR images. In this study, we utilize simultaneously both shortaxis and long-axis MR volumes. The SA and LA volumes are first transformed into same coordinate system using image header information. Then, the segmentation takes place in this coordinate system. The use of two volumes provides supplementary information that enables more accurate segmentation of the ventricles in the basal and apical levels.

In this paper, we apply a deformable model-based segmentation method in this context. A mean shape and grey-level model, which is constructed from a database, is deformed using deformation spheres [12. Previously, we have used similar framework in the tracking of cardiac MR images [13, and in the construction of statistical shape model [14]. Lelieveldt et al. constructed an AAM from three MR views, and used the model to segment the left ventricle simultaneously from all three views in 15 . However, to our knowledge, the simultaneous segmentation of the ventricles and atria from SA and LA MR volumes has not been reported earlier.

\section{Material}

The cardiac MR volumes of 25 healthy control persons of two separate clinical studies formed the database of this study. The volumes were obtained with 1.5 T Siemens Vision and Siemens Sonata MR devises with a phased array coil (Siemens, Erlangen, Germany) at the Helsinki Medical Imaging Center in the University of Helsinki. A standard turboflash cine imaging series was obtained with ECG-gating during a breath hold. SA volumes contained ventricles from the valve level until the last level where the apex is still visible, and LA volumes contained atria and ventricles. The pixel size of both SA and LA image slices was either $1.0 \times 1.0 \mathrm{~mm}$ or $1.4 \times 1.4 \mathrm{~mm}$, and the slice thickness was $6-7 \mathrm{~mm}$. The number of slices in the SA volumes was $4-5$, and in the LA volumes $4-7$. Examples of the SA and LA image slices are given in Fig. 1 for one database subject. The ventricles, atria, and epicardium were manually segmented from the volumes by an expert. Thereafter, triangle surface models were constructed from the manual segmentations (Fig. 1 1 ). 


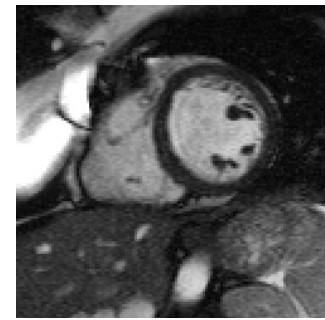

(a)

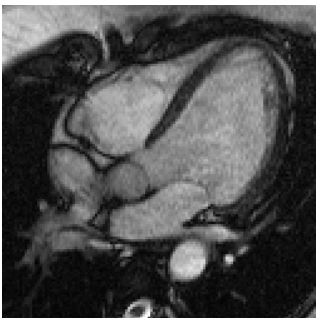

(b)

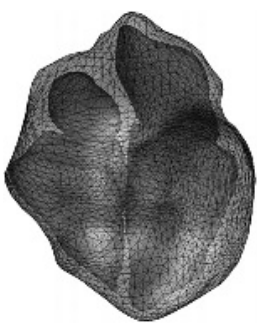

(c)

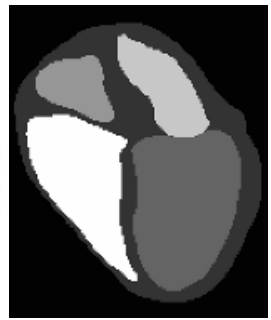

(d)

Fig. 1. a) A short-axis, b) long-axis slice, and c) a triangle surface model of one database subject. d) A slice from a labeled volume constructed from the manual segmentation

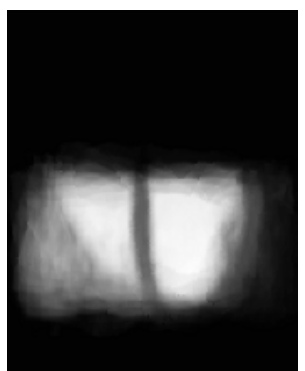

(a)

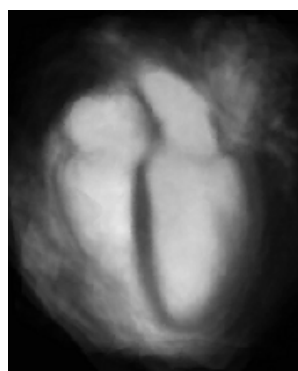

(b)

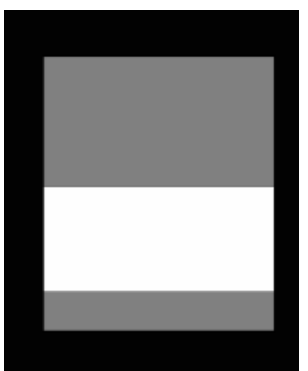

(c)

Fig. 2. a) A short-axis, b) long-axis mean model. c) A mask used in the calculation of the similarity measure

\subsection{Mean Shape and Grey-Level Model}

A mean shape and grey-level model (Fig. 2) was constructed from the database using the procedure presented in [16. One subject was randomly selected as a reference. The remaining database volumes were registered with the reference volume using translation, rotation, and isotropic scaling. The registration method used for this purpose is presented in Section 3.3 Next, the reference volume was non-rigidly registered to the database volumes. The non-rigid registration method based on deformation spheres was used in this step [12. Labeled volumes constructed from the manual segmentations (Fig.11d) were used instead of the real grey-level volumes to ensure accurate registrations of the object surfaces [17. From the resulting deformation fields, a mean deformation field was computed, and it was applied to the reference volume. This produced a mean shape model. To end up with a mean grey-level volume, the deformation fields of the non-rigid registrations were used to register the database volumes to the mean shape model, and the grey-level values were averaged voxel-wise. This procedure was repeated twice by using the obtained mean model as the reference to reduce the bias of the mean shape towards the selected reference subject. 


\section{Methods}

The basic idea of the segmentation and registration methods studied in this paper is to deform an a priori model to match with the target volume. The deformations are determined based on a voxel similarity measure that is computed from both the SA and LA volumes. The mean model that was constructed in Section 2.1 is used as an a priori model.

\subsection{Preprocessing}

Because both the SA and LA volumes are used simultaneously in segmentation, the correspondence between the SA and LA volumes has to be determined. The necessary information is available in the image headers. Based on this information, the translation, rotation, and scaling parameters that transform the LA volumes to the coordinate system of the SA volumes, or vice versa, can be computed [13. In this study, this procedure is used to transform the LA volumes into the coordinate system of the SA volumes.

There are several sources of motion artifacts (e.g., breathing) in the cine cardiac MR imaging. Consequently, the slices may be misaligned, and the correspondence between the SA and LA volumes may be inaccurate. The motion artifacts are corrected from the data using a registration-based method that moves the slices, and optimizes the normalized mutual information (NMI) between the SA and LA volumes [18]. After this, the voxel-by-voxel correspondence between the SA and LA volumes is guaranteed.

\subsection{Similarity Measure}

The initial alignment and image segmentation are implemented using normalized mutual information (NMI) as a similarity measure [19]. The NMI is defined as

$$
I\left(S, T_{i}\right)=\frac{H(S)+H\left(T_{i}\right)}{H\left(S, T_{i}\right)},
$$

where $H(S)$ and $H\left(T_{i}\right)$ are the marginal entropies and $H\left(S, T_{i}\right)$ is the joint entropy of the source data $S$ and the target data $T_{i}$.

In this study, the source data consist of both SA and LA source data, $S=$ $\left\{S_{S A}, S_{L A}\right\}$, and the target data consist of the SA and LA data of the target subject, $T_{i}=\left\{T_{i, S A}, T_{i, L A}\right\}$. For example, the histogram that is used to calculate the joint entropy $H\left(S, T_{i}\right)$ is built from both the SA and LA volumes. The volume in which the SA source volume has meaningful information constitutes the SA source data, $S_{S A}$. Similarly, a part of the LA source volume constitutes the LA source data, $S_{L A}$. A mask is made to determine these regions (Fig. 25). The SA volume information is used in the white regions, and the LA volume information in both the white and grey regions of Fig. 2r. 


\subsection{Affine Registration}

The transformation parameters for the initial alignment of the database volumes with the mean model are optimized using the similarity measure presented in Section 3.2 and the Simplex optimization algorithm. Both seven- (rigid plus isotropic scaling) and nine-parameter (rigid plus anisotropic scaling) affine transformations were studied. The seven-parameter affine transformation was selected because it proved to be more robust and accurate than the nine-parameter affine transformation.

\subsection{Non-rigid Registration-Based Segmentation}

The mean model and the affinely registered database volumes are used as source and target volumes, respectively, in a deformable model-based segmentation method. The method registers non-rigidly the mean model to the database volumes using deformation spheres [12. In this method, smooth deformations are applied to the voxels inside a sphere in such a way that the NMI (Section 3.2) is maximized. The location of the sphere is randomly chosen from the surfaces of the ventricles, atria, and epicardium, and it is varied during the iteration. The radius of the sphere is iteratively decreased from 30 voxels to 10 voxels. The deformation can be regularized in several ways in the segmentation tool. In this study, the directions of the normal vectors of the surfaces are regularized.

\subsection{Evaluation Methods}

The registration and segmentation methods presented in this section are evaluated using the database presented in Section 2 Leave-one-out cross-validation is used: each database subject is once regarded as a target, and the mean model used as an a priori model is constructed from the remaining 24 database subjects. For comparison, identical segmentations were performed using only the SA volumes.

The segmentation/registration error is defined as the mean distance from the manually segmented target surface to the deformed mean model's surface:

$$
E \equiv \frac{1}{N_{t}} \sum_{i=1}^{N_{t}} d\left(\mathbf{t}_{i}, S\right),
$$

where $N_{t}$ is the number of the nodes in the target triangle surface model, $\mathbf{t}_{i}$ is the $i$ th target node, and $d\left(\mathbf{t}_{i}, S\right)$ is the Euclidean distance from the point $\mathbf{t}_{i}$ to the triangle surface of the deformed mean model, $S$. The error of the epicardium is computed only from the surface below the valve level.

\section{Results}

The results for the segmentation using only the SA volumes and for the segmentation using both the SA and LA volumes are given in Table 1. For one 
Table 1. The segmentation/registration errors (mean \pm standard deviation) after the affine registration and after the non-rigid registration-based segmentation

\begin{tabular}{|c|c|c|c|c|}
\hline & \multicolumn{2}{|c|}{$\begin{array}{l}E(\mathrm{~mm}) \\
\text { after affine registration }\end{array}$} & \multicolumn{2}{|c|}{$\begin{array}{c}E(\mathrm{~mm}) \\
\text { after segmentation }\end{array}$} \\
\hline organ & only SA & $\mathrm{SA}+\mathrm{LA}$ & only SA & $\mathrm{SA}+\mathrm{LA}$ \\
\hline LV & $4.20 \pm 1.64$ & $2.97 \pm 0.77$ & $3.12 \pm 1.40$ & $2.62 \pm 0.75$ \\
\hline RV & $4.84 \pm 2.06$ & $3.75 \pm 1.01$ & $4.13 \pm 2.68$ & $3.66 \pm 1.04$ \\
\hline LA & $5.71 \pm 2.50$ & $3.26 \pm 1.34$ & & $2.62 \pm 1.11$ \\
\hline RA & $7.14 \pm 4.15$ & $3.76 \pm 1.08$ & & $2.85 \pm 0.89$ \\
\hline epicardium & $4.38 \pm 1.88$ & $3.30 \pm 0.72$ & $3.62 \pm 1.46$ & $3.21 \pm 0.65$ \\
\hline all & $5.25 \pm 2.22$ & $3.41 \pm 0.66$ & & $2.99 \pm 0.58$ \\
\hline
\end{tabular}
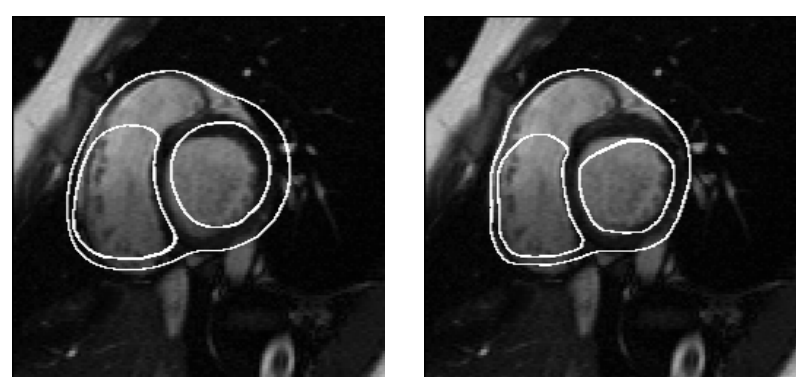

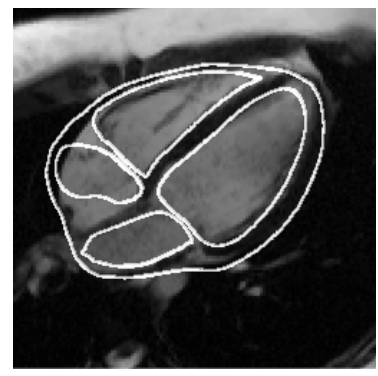

(a)

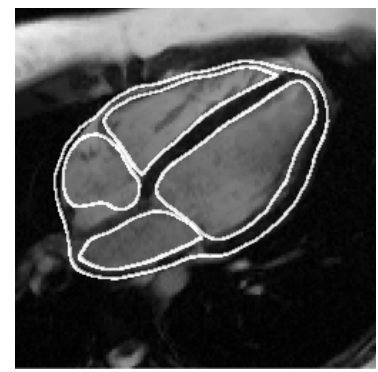

(b)

Fig. 3. An SA and LA image slice of the results for one database subject a) after the affine registration $(E=3.83 \mathrm{~mm})$, and b) after the non-rigid registration-based segmentation $(E=2.96 \mathrm{~mm})$

database subject, the method using only the SA volumes produced totally incorrect segmentation. To enable reasonable comparison, this database subject was excluded from the results presented in Table 1 Therefore, the error values in Table 1 are the mean errors of 24 target subjects. The removed database subject gave the worst accuracy also for the segmentation using both the SA and LA volumes. When all 25 database subjects were used as a target, the mean segmentation error of all organs was $E=3.09 \pm 0.75 \mathrm{~mm}$. The results for one database subject after the affine registration and the non-rigid registration-based segmentation are shown in Fig. 3 . 
The segmentation using both the SA and LA volumes was performed also by using a randomly selected database subject as an a priori model instead of the mean model. The mean errors of all organs, which are comparable to the results in Table 1 were $E=5.72 \pm 2.32$ after the affine registration and $E=4.22 \pm 1.83$ after the non-rigid registration-based segmentation.

\section{Discussion}

The results in Table 1 showed that by utilizing both the short-axis and longaxis MR volumes the ventricles and atria can be simultaneously segmented. Especially, the segmentation accuracy of the atria was improved from the affine registration. The superiority of using both the SA and LA volumes instead of just the SA volumes was demonstrated. The reason for the improved segmentation accuracy is that the comprehensive information on the SA and LA volumes is utilized to produce more accurate spatial transformations. In addition, the LA volumes include areas which are not visible in the SA volumes. It is worth of noting that the SA volumes used in this study did not contain atria. Therefore, when only the SA volumes were used in the affine registration, the transformation parameters had to be estimated from the ventricle data only. Furthermore, this is why the segmentation errors in Table 1 are reported only for the ventricles and epicardium when only the SA volumes were used.

We also proved that better accuracy is obtained by using a mean model as an a priori model instead of a randomly selected database subject. Naturally, if another database subject had been selected, the results could have been different.

In this study, the LA volumes were transformed into the coordinate system of the SA volumes (Section 3.1), in which the segmentation was performed. When the LA volumes were transformed into the coordinate system of the SA volumes, interpolation errors were produced. The registration and segmentation could have been performed with the LA volumes in their own coordinate system, but this would have increased the computational complexity.

Acknowledgements. Research was supported by Tekes, the National Technology Agency, Finland, the Finnish Cultural Foundation, and the Foundation for Advancement of Technical Sciences, Finland.

\section{References}

1. A.F. Frangi, W.J. Niessen, M.A. Viergever. Three-Dimensional Modeling for Functional Analysis of Cardiac Images: A Review. IEEE Trans. Med. Imag., vol. 20(1), pp. 2-25, 2001.

2. M. Lorenzo-Valdés, G.I. Sanchez-Ortiz, R. Mohiaddin, D. Rueckert. Atlas-Based Segmentation and Tracking of 3D Cardiac MR Images Using Non-rigid Registration. Proc. MICCAI'O2, 642-650, 2002.

3. M. Lorenzo-Valdés, G.I. Sanchez-Ortiz, R. Mohiaddin, D. Rueckert. Segmentation of 4D Cardiac MR Images Using a Probabilistic Atlas and the EM Algorithm. Proc. MICCAI'03, 440-450, 2003. 
4. T.F. Cootes, C.J. Taylor, D.H. Cooper, J. Graham. Active Shape Models - their training and application. Comput. Vis. Image Underst., vol. 61(1), 38-59, 1995.

5. T.F. Cootes, G.J. Edwards, C.J. Taylor. Active Appearance Models. Proc. European Conference on Computer Vision, 484-498, 1998.

6. M.R. Kaus, J. von Berg, W. Niessen, V. Pekar. Automated Segmentation of the Left Ventricle in Cardiac MRI. Proc. MICCAI'03, 432-439, 2003.

7. S.C. Mitchell, B.P.F. Lelieveldt, R.J. van der Geest, H.G. Bosch, J.H.C. Reiber, M. Sonka. Multistage Hybrid Active Appearance Model Matching: Segmentation of Left and Right Ventricles in Cardiac MR Images. IEEE Trans. Med. Imag., vol. 20(5), pp. 415-423, 2001.

8. S.C. Mitchell, J.G. Bosch, B.P.F. Lelieveldt, R.J. van der Geest, J.H.C. Reiber, M. Sonka. 3-D Active Appearance Models: Segmentation of Cardiac MR and Ultrasound Images. IEEE Trans. Med. Imag., vol. 21(9), pp. 1167-1178, 2002.

9. J.S. Suri. Computer Vision, Pattern Recognition and Image Processing in Left Ventricle Segmentation: The Last 50 Years. Pattern Analysis \& Applications, vol. 3, 209-242, 2000.

10. N. Paragios. A Level Set Approach for Shape-Driven Segmentation and Tracking of the Left Ventricle. IEEE Trans. Med. Imag., vol. 22(6), pp. 773-776, 2003.

11. D.T. Gering. Automatic Segmentation of Cardiac MRI. Proc. MICCAI'03, 524532, 2003.

12. J. Lötjönen, T. Mäkelä. Elastic matching using a deformation sphere. Proc. MICCAI'01, 541-548, 2001.

13. J. Lötjönen, D. Smutek, S. Kivistö, K. Lauerma. Tracking Atria and Ventricles Simultaneously from Cardiac Short- and Long-Axis MR Images. Proc. MICCAI'03, 467-474, 2003.

14. J. Lötjönen, J. Koikkalainen, D. Smutek, S. Kivistö, K. Lauerma. Four-Chamber 3-D Statistical Shape Model from Cardiac Short-Axis and Long-Axis MR Images. Proc. MICCAI'03, 459-466, 2003.

15. B.P.F. Lelieveldt, M. Üzümcü, R.J. van der Geest, J.H.C. Reiber, M. Sonka. Multiview Active Appearance Models for Consistent Segmentation of Multiple Standard Views: Application to Long and Short-axis Cardiac MR Images. Computer Assisted Radiology and Surgery, vol. 1256, 1141-1146, 2003.

16. A. Guimond, J-P. Thirion. Average Brain Models. A Convergence Study. Computer Vision and Image Understanding, vol. 2, 192-210, 2000.

17. A.J. Frangi, D. Rueckert, J.A. Schnabel, W.J. Niessen. Automatic Construction of Multiple-Object Three-Dimensional Statistical Shape Models: Application to Cardiac Modeling. IEEE Trans. Med. Imag., vol. 21(9), pp. 1151-1166, 2002.

18. J. Lötjönen, M. Pollari, S. Kivistö, K. Lauerma. Correction of movement artifacts from 4-D cardiac short- and long-axis MR data. Proc. MICCAI'04, 2004. In press.

19. C. Studholme, D.L.G. Hill, D.J. Hawkes. Automated three-dimensional registration of magnetic resonance and positron emission tomography brain images by multiresolution optimization of voxel similarity measures. Medical Physics, vol 24(1), 71-86, 1997. 